\title{
2006-924: AVOIDING FINITE ELEMENT ANALYSIS ERRORS
}

Joseph Dues, Purdue University-New Albany 


\title{
Avoiding Finite Element Analysis Errors
}

\begin{abstract}
Finite Element Analysis (FEA) is a numerical method for calculating stress and strain (and other quantities) in structures that cannot be easily analyzed any other way. FEA Analysts use complex software to create a mathematic representation of the physical structure being studied, apply loads to the structure and then solve for the resulting displacements and stresses. In years past, FEA was performed by highly trained analysts with master's level engineering degrees or higher. Today, the combination of competitive market pressures, powerful computer hardware and well designed software has resulted in CAD designers being asked to perform FEA early in the design process. To enable these designers to perform FEA analysis, solid modeling software vendors have incorporated FEA into their solid modeling and design drafting packages. Unfortunately, while the software is easy to use, CAD designers usually do not have the education in specialized mechanics and high level mathematics necessary to understand the calculations being performed by the FEA software. Even more important, without special training, they do not have the ability to recognize the potential for errors in the FEA results. Errors may occur during the modeling and analysis process due to limitations of the software as well as user error. Understanding and avoiding these errors dramatically improves the chances the designer will successfully utilize FEA software.
\end{abstract}

Autodesk Inventor Professional 9 now includes FEA capability from ANSYS, Inc. Earlier versions of this software (without FEA) were used at Purdue University - College of Technology at New Albany (PUNA) to instruct students in solid modeling and design drafting. With new and ever expanding software capabilities, CAD instructors had to determine how to prepare engineering technology students to use to software appropriately. Today, after students learn to use the software, they are given assignments that demonstrate the potential weaknesses of FEA software. This paper discusses the assignments and compares simple problems solved in Inventor to exact results to show how simple mistakes result in poor FEA results. It also includes a discussion of the implementation of FEA in Inventor and strategies for educating engineering technology students in FEA.

Introduction

Finite Element Analysis (FEA) is a method for solving the equations that describe the behavior of elastic solids when subject to loads. Finite Element software that employs this procedure allows engineers to calculate stress and strain in objects that cannot be easily analyzed by manual calculations. The FE method was first described by R. Courant in 1943 to solve a torsion problem but it failed to gain widespread use due to the volume of calculations needed. ${ }^{1}$ FEA requires an extremely large number of calculations to solve and is only practical today due to modern advances in computer speed and capacity. In the 1970's, general purpose finite element software was developed due to the increasing availability and power of digital computers. Digital computers in the form of mainframe computers provided an efficient tool to perform finite element calculations. Since then, computer hardware has rapidly increased in speed and storage capacity and the FEA software has gained better interfaces, pre and post processing of the data and improved graphics. ${ }^{2}$ 
Since the early days of FEA, there has been a tremendous increase in computer speed and capacity. The development of CAD software to document designs and Finite Element Analysis software to verify designs has reduced the need for physical prototypes. In addition, since the 1990's there has been an increased use of 3D modeling and the combination of CAD, 3D Modeling and Finite Element Analysis into a single package. All of this has lead to an increased reliance on virtual prototypes instead of physical prototypes.

Likewise, the personnel involved with FEA have changed over the years. Initially, the personnel typically included a drafter (or drafters) and an analyst. The drafters would create and document a design and then pass the design on to the analyst. The analyst would then begin an evaluation of the design. Usually the analyst would have to recreate the geometry of the design since the CAD software used to document the design often was not compatible with the software used to analyze the design. The analyst, after inputting the geometry, then used the FEA software to evaluate the design. More often than not, the FEA results would indicate that the design could use some improvements. The analyst would then pass the revisions back to the drafter to be reworked. Clearly this process could consume a considerable amount of time before a final design was reached.

Modern companies are forced by competition in the marketplace to reduce the time it takes to develop a new product. Improving the efficiency of the analysis process is one way to decrease the time to market. To address this, companies now perform analysis as early in the design process a possible. This means that modern designers must perform some analysis concurrently with the product design. The downside to early use of FEA in the design cycle is that the designer may not be as thoroughly trained as the traditional analysts (see Table 1). Thus there is an increased risk of errors or omissions in the analysis.

\begin{tabular}{|l|l|l|l|}
\hline & Traditional Drafter & Traditional Analysts & Modern Designer \\
\hline Education & $\begin{array}{l}\text { Technical Associate } \\
\text { Degree }\end{array}$ & $\begin{array}{l}\text { Engineering Masters } \\
\text { or Doctorate }\end{array}$ & $\begin{array}{l}\text { Technical or } \\
\text { Engineering } \\
\text { Bachelors Degree }\end{array}$ \\
\hline Mechanics Exposure & $\begin{array}{l}\text { Little or No } \\
\text { Mechanics }\end{array}$ & $\begin{array}{l}\text { Specialized } \\
\text { Mechanics Education }\end{array}$ & Basic Mechanics \\
\hline $\begin{array}{l}\text { Exposure } \\
\text { Design } \\
\text { Documentation }\end{array}$ & Trigonometry & $\begin{array}{l}\text { High Level } \\
\text { Mathematics }\end{array}$ & $\begin{array}{l}\text { Calculus (1 or } 2 \\
\text { semesters) }\end{array}$ \\
\hline
\end{tabular}

Table 1. FEA Personnel.

Competitive pressures, powerful computer hardware and well designed software have lead to designers being asked to perform equipment analysis earlier in the design cycle. As educators, how do we prepare the modern designer for this role? In addition, as professionals, what do we need to know about analysis capability of modern software? This paper describes 3 examples 
used in the classroom during an introduction to FEA in a 3D modeling class. These examples illustrate some of the situations the designer should watch out for when using FEA.

\section{FEA Procedure}

A general FEA procedure starts with a definition of the problem's geometry with solid model. The solid model may be created using pre-processing software bundled with FEA program or it may be generated using a popular 3D modeling package and then imported into the FEA program. The second step is to define the constraints on the solid model. Usually these are the locations on the model where the object is restrained from displacement and/or rotation. Displacement constraints prevent movement in any or all directions in 3 dimensional space. Likewise, rotational constraints prevent movement of the model around any or all axes in space. The next task is to apply the expected loads on the model. The simplest type of load is concentrated forces: singular forces in a particular direction applied to a discrete point. Other types of loads include pressure applied to a surface and body loads (such as gravity) that are applied to the entire volume of the model. Once the geometry is defined and the constraints and loads applied, the model is divided into finite elements. This process splits the solid model into many small blocks called elements. Each element is connected to adjacent elements at nodes. This process of often called "meshing" because the resulting graphical display of the solid model looks like a mesh has been laid over the object being analyzed. The size of the elements and the numbers of nodes is dependent on the software and the options selected by the user. The model is then put through the solution routines and the results are calculated. During the solution procedure, each element is evaluated as a small part of the whole and the overall solution is found from the individual effect of each element. Finally, the results are evaluated with postprocessing software, usually by displaying the stresses in the part in the graphical form of a color contour plot. ${ }^{1}$

\section{FEA using Inventor}

Inventor Professional 9 is the latest version of 3D modeling software from Autodesk, Inc., San Rafael, CA. Inventor now includes stress analysis capability by including FEA software from ANSYS, Inc., Canonsburg, PA. Inventor can now perform linear stress analysis on structures where there is no yielding, only small deformations and no temperature effects. While this capability is only a small portion of the types of analysis full fledged FEA packages offer, it's sufficient for many types of initial analyses.

Each FEA solution is a tradeoff between the most accurate possible solution by maximizing the number of nodes and the efficient use of computer resources by limiting the number of elements. Increasing the number of nodes in a model dramatically increases the amount of time it takes to solve the model. Even on modern PC's, large models can take hours to perform the calculations needed to find the solution. For this reason, most analysts start with a model with only a moderate number of nodes and gradually refine the mesh to improve the results. There are two types of refinement: H-refinement and P-refinement. In H-refinement, the user increases the number of elements and decreases the element size without changing the element type in order to improve the FEA results. P-refinement attempts to improve analysis results by keeping the element size constant but increasing the number of nodes by changing the element type to an element that has more nodes per element. 
Autodesk Inventor Professional 9 uses H-refinement to improve analysis results. Inventor has a "Mesh Relevance" control that allows the user to modify the mesh. Setting mesh relevance to 100 results in a relatively coarse mesh. An average mesh is generated when mesh relevance is set to 0 and a fine mesh when it's set to +100 . Inventor uses the mesh relevance setting to adjust the element size. Autodesk explains that after mesh relevance is set, element size is based on the "Size of the body box, body curvature and the complexity of features." 3 The overall result is that the user has little direct control of the mesh.

\section{Illustrative Examples}

The following examples show how stress results calculated using the analysis procedures in Inventor compare to theoretical results from standard strength of materials calculations. Before analyzing the cantilever test examples in Inventor, the stress at the areas of interest for each beam was calculated manually. These manual calculations provide the theoretical values of stress that the results from Inventor can be compared to.

The three test examples were modeled in Inventor and then analyzed using its stress analysis module. The nodes on the fixed end of the cantilever beams were restrained from motion in all six degrees of freedom. In all three examples, the fixed side of the beam is on the left in the diagrams. The concentrated force was applied to the opposite end of the beam acting downward. After the analysis is complete, Inventor displayed a color plot showing the stress distribution in the analyzed part (see Figures 2, 4 and 7). The user can then query the results to find the exact level of stress calculated by Inventor for particular areas of interest. These stress values are reported in Tables 2, 3 and 4 and compared to the theoretical stress values.

The first test example is a cantilever beam with a fixed support at one end and a $150 \mathrm{lbf}$. load at the other (see Figure 1). It's 10 inches long, 1 inch tall, and 0.25 inch wide with a 0.75 inch diameter hole 3 inches from the supported end. The purpose of this example is to test how well Inventor models the stress concentration that the transverse hole creates. A 0.75 inch hole in a 1 inch tall beam creates a stress concentration of about 1.5 times the nominal stress in the beam at the location of the hole.

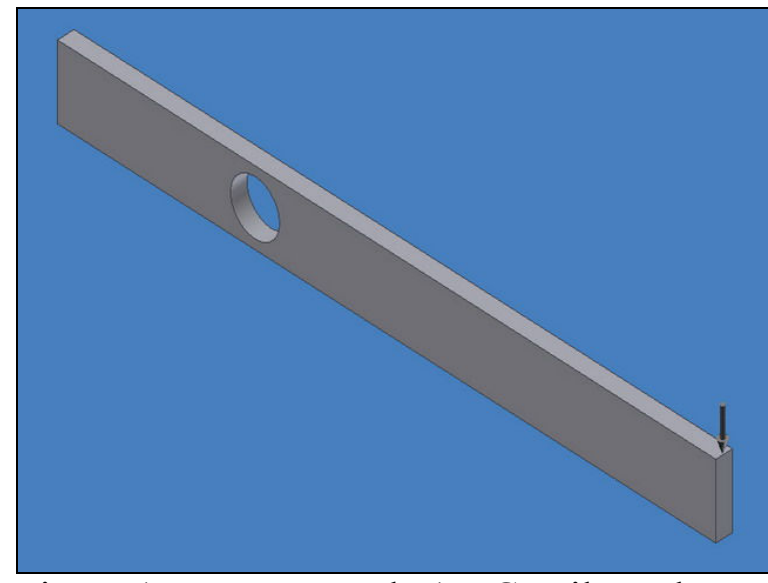

Figure 1. Test Example 1 - Cantilever beam.

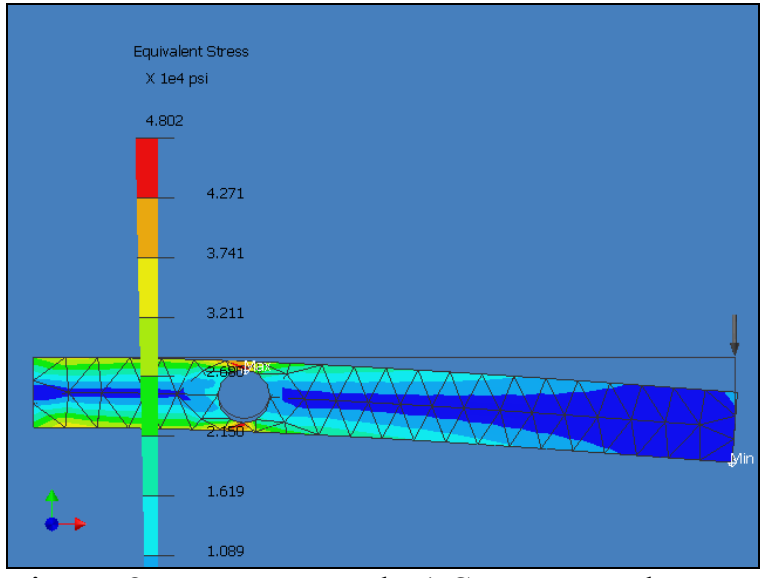

Figure 2. Test Example 1 Stress Results. 


\begin{tabular}{|c|c|c|c|c|c|}
\hline Description & No. of Nodes & Stress @ Hole & Percent Error & $\begin{array}{c}\text { Stress @ } \\
\text { Support }\end{array}$ & Percent Error \\
\hline Theory & - & $37800 \mathrm{psi}$ & - & $36000 \mathrm{psi}$ & - \\
\hline Coarse Mesh & 380 & $38000 \mathrm{psi}$ & $+0.5 \%$ & $33000 \mathrm{psi}$ & $-8.3 \%$ \\
\hline Normal Mesh & 781 & $37400 \mathrm{psi}$ & $-1.0 \%$ & $32000 \mathrm{psi}$ & $-11.1 \%$ \\
\hline Fine Mesh & 963 & $38800 \mathrm{psi}$ & $+2.7 \%$ & $32500 \mathrm{psi}$ & $-9.7 \%$ \\
\hline
\end{tabular}

Table 2. Results for Test Example 1 - Cantilever with transverse hole.

In Test Example 1, the hole in the beam creates a stress concentration and the Inventor results match very well with theory; less than $3 \%$ difference regardless of the mesh. Likewise, at the supports, the results did not change much as the mesh was changed from a coarse mesh to a fine mesh. There was however, slightly more difference between the theoretical value for the stress and the Inventor results. Inventor calculated stress levels at the supports from $8 \%$ to $11 \%$ less than that predicted by theory. This may be explained by the displacement constraints applied to restrain this end of the beam. The displacement constraints may be artificially reinforcing the beam, thus causing Inventor to calculate lower levels of stress. The results from Test Example 1 show that the effects of a hole is well represented in this model and that changing the mesh relevance doesn't have much of an effect.

The second test example is a cantilever beam with a step change in height. The stepped plate has a sharp inside corner at the change in height. The main part of the cantilever beam is 10 inches long, 1 inch tall and 1/4 inch wide (see Figure 3). After the step change, the beam is 5 inches tall.

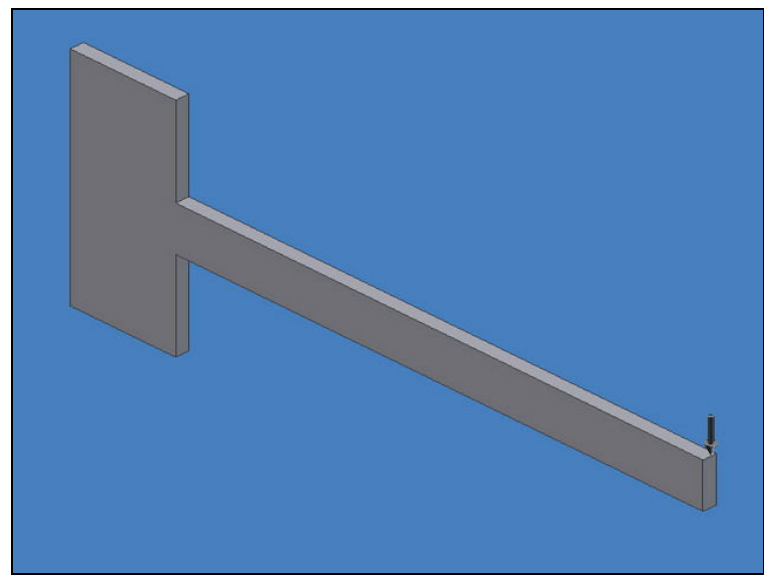

Figure 3. Test Example 2 - Stepped Plate.

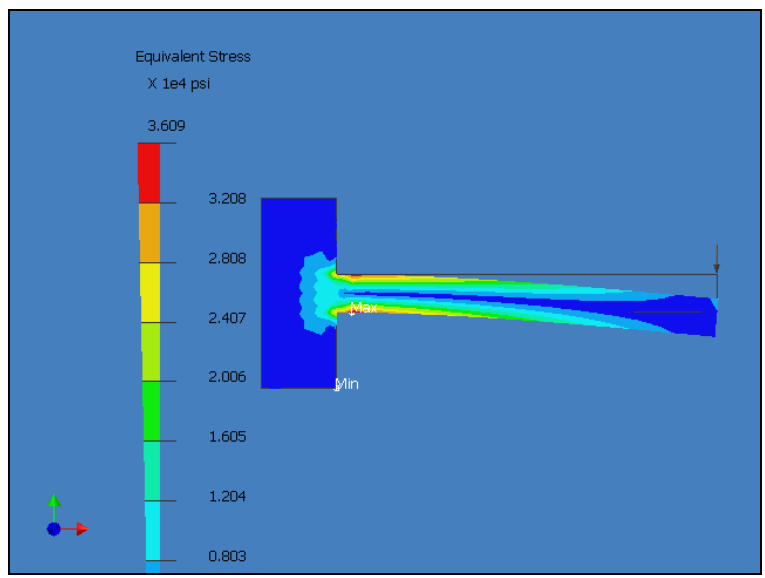

Figure 4. Stress Results for Test Example 2. 


\begin{tabular}{|c|c|c|c|}
\hline Description & No. of Nodes & Stress @ Support & Percent Error \\
\hline $\begin{array}{c}\text { Theory (with stress } \\
\text { concentrator) }\end{array}$ & - & $93600 \mathrm{psi}$ & - \\
\hline $\begin{array}{c}\text { Inventor Stress } \\
\text { Analysis }\end{array}$ & 1198 & $36100 \mathrm{psi}$ & $-61.4 \%$ \\
\hline
\end{tabular}

Table 3. Results for Test Example 2 - Cantilever with sharp step change.

A severe test for FEA software is the sharp inside corner as shown in example 2. If the student models the cantilever beam without a fillet, then the resulting stress calculated by Inventor is close to the stress that exists if there is no stress concentration at all (36000 psi in this example). The danger of this is twofold; first, if the student is really intending to have a very sharp inside corner, then Inventor reports a stress level that is two to three times less that the actual expected stress level. The student could easily believe that the design was acceptable when it is not. Second, if the student really is intending to put a small fillet in the corner, he may be lead into believing that the fillet is less important than it really is since the stress levels in the corner are not very high.

Inventor calculated a stress level of 36,100 psi for test example 2 . This is only $1 / 3$ of the stress level expected using a stress concentration factor of 2.6 for the sharp corner. Since the stress concentration factor could conceivably be higher, this is a very large discrepancy between Inventor results and the expected stress levels in the actual part.

In addition to the problems with the stress levels, the novice user may also be misled by the way Inventor displays the results for this problem. Close inspection of the Equivalent Stress plot (Figure 4) shows that the maximum stress is a short distance away from the step change, not in the corner as expected. This is a result of the stress averaging that Inventor does when it is plotting the stress results. Since the nodes in the corner are attached to both an element in the tall portion of the beam (low stress) and an element in the short part of the beam (high stress) then the average stress for that node is reported to be lower than the stress for nodes attached to only elements in the short portion of the beam. The effect of this is that the maximum displayed stress is several elements removed from the corner. Depending on element size, this effect may be very misleading to the student, again indicating that stress levels in the corner are not as severe as they should be.

Test Example 3 (Figure 5) is also a cantilever beam with a step change, but this time there is a 0.05 in filleted corner at the step change in height. The main part of the cantilever beam is still 10 inches long, 1 inch tall and 0.25 inches wide. After the step change, the beam is 5 inches tall. 


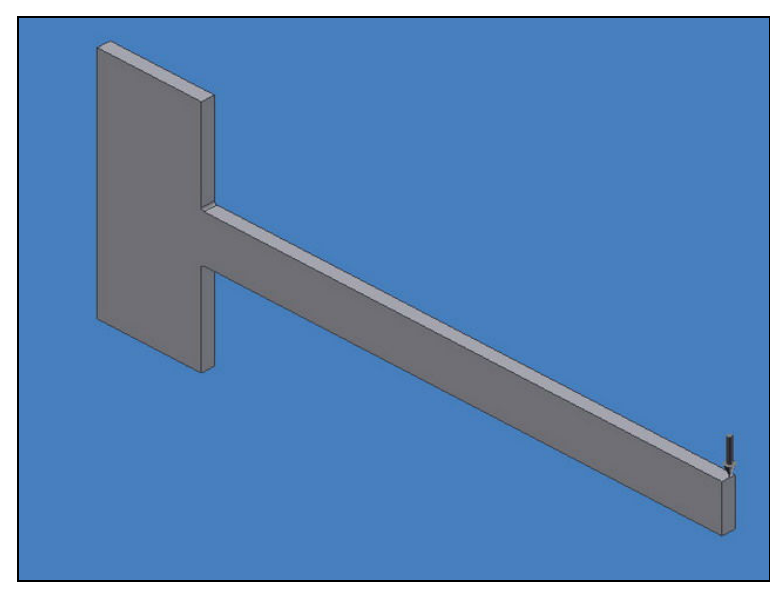

Figure 5. Test Example 3 - Stepped Plate with fillet.

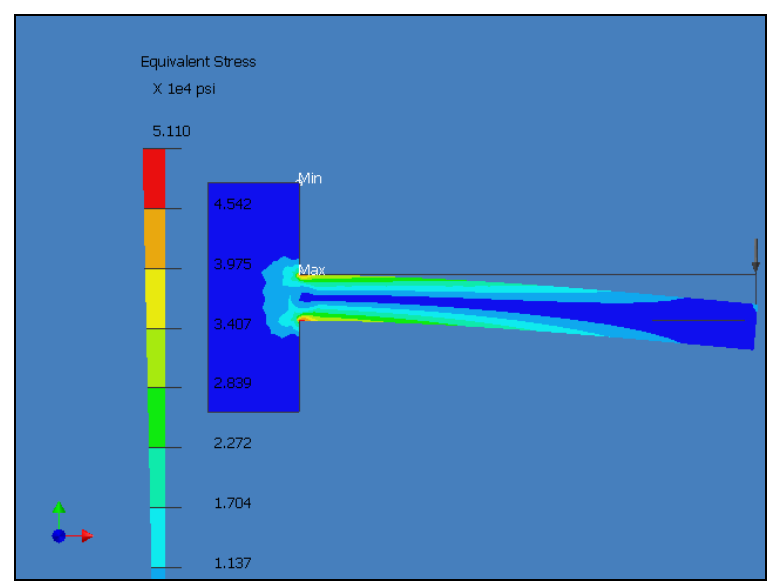

Figure 7. Stress result for Example 3.

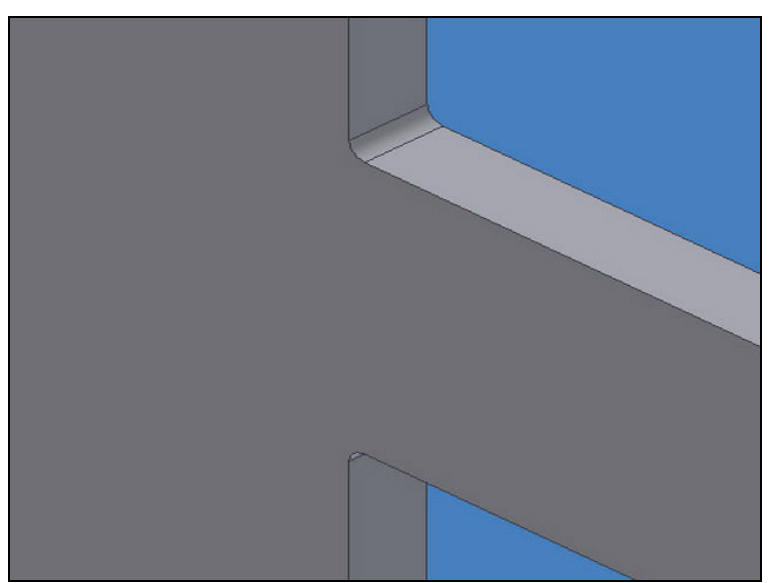

Figure 6. Close-up of filleted step change.

\begin{tabular}{|c|c|c|c|}
\hline Description & No. of Nodes & Stress @ Support & Percent Error \\
\hline $\begin{array}{c}\text { With Stress } \\
\text { Concentrator }\end{array}$ & - & $93600 \mathrm{psi}$ & - \\
\hline $\begin{array}{c}\text { Inventor Stress } \\
\text { Analsyis (Fine) }\end{array}$ & 1198 & $51100 \mathrm{psi}$ & $-45.4 \%$ \\
\hline
\end{tabular}

Table 4. Results for Test Example 3 - Cantilever with filleted step change.

To address the problems found in example 2, Test Example 3 has a 0.05 inch fillet added to the corner of the step change on the cantilever beam (see Figure 6). For the FEA mesh, the added fillet had the effect of forcing Inventor to use a smaller element size in that area. This caused the calculated stress to jump $42 \%$ to 51,100 psi (see Table 4). While this is an improvement, it's still considerably below the theoretical stress of 93,600 psi for a stepped plate with a fillet of this size. However, the addition of a fillet did resolve the problem with the misplaced maximum 
stress. In Figure 7, the location of the maximum stress is now in the corner of the step where is should be. Example 3 shows the importance of including fillets of inside corners in the FEA model.

Inventor in the Classroom

The previous three examples are first given to the students to analyze using Inventor. The students are then given the theoretical stress levels and have to compare the theoretical stress to their results. Since this exercise is part of a CAD class early in their education, most students have not taken a strength of materials class. Thus they are similar to may drafters in lacking much engineering mechanics knowledge. Once the students see the potentially significant difference between Inventor and theory in certain circumstances, the instructor leads a discussion to reinforce the following points about using FEA:

- FEA is a mathematical model of physical reality.

- Assumptions are made to simplify the FE model, but the user needs to understand the effects of each assumption.

- The physical problem may not be completely defined or understood.

- Discrete elements are used to represent a continuous physical problem discretization error can be reduced by refining the mesh.

- The Ideal is an infinite mesh - but this would require infinite computing power.

- Small mistakes or omissions can make answers completely wrong.

- Students must examine their FEA results with a critical eye.

- Continuing education is critical to effectively utilize new software features.

This exercise shows that non-specialists can operate analysis software without understanding the calculations needed to arrive at a solution manually. However, small mistakes or a misunderstanding of analysis limits can make the answers very misleading or even completely wrong. These analysis errors can be reduced by education, limiting user options and advanced, automatic controls and reports. Inventor provides a limited amount of analysis capability suitable for designers with the appropriate skills but ultimately gives the user very little control over the analysis process.

Bibliography

[1] Finite Element Modeling for Stress Analysis, Robert D. Cook, 1995, John Wiley and Sons, Inc., New York, NY

[2] "Testing and Evaluation of Errors Associated with Finite Element Models used in Design Analysis", Joseph F Dues, Jr., 1997, University of Louisville

[3] "Stress Analysis Getting Started", Autodesk Inventor Professional 9, 2004, Autodesk, Inc., San Rafael, CA 